\title{
Immunochromatographic Assessment of Salivary Cotinine and Its Correlation With Nicotine Dependence in Tobacco Chewers
}

ORIGINAL

ARTICLE

\author{
Asha V, Dhanya M \\ Department of Oral Medicine and Radiology, The Oxford Dental College and Hospital, Bengaluru, India
}

\begin{abstract}
Background: This study assessed the correlation between nicotine dependence and salivary cotinine levels in tobacco chewers and checked the reliability of Fagerstorm test in tobacco cessation programmes.

Methods: The study sample included 75 tobacco chewers aged between 20 to 50 years. Self-reported nicotine dependence was evaluated using Fagerstorm Test for Nicotine dependence-smokeless tobacco questionnaire. Patients were categorized into low, moderate and high dependent chewers based on their answers to the questionnaire. The unstimulated salivary cotinine levels were measured by immunochromatographic assay using the NicAlert saliva test. Data was analysed using the chi-square test for testing intra-group variation, analysis of variance for testing between-groups variation, and the Spearman coefficient for assessing the association between variables.

Results: No statistically significant association was seen between salivary cotinine levels and nicotine dependence. A weak positive correlation was noted between the above variables $(r=0.230)$. In the group studied, it was evident that the patients were under-reporting the nicotine dependence.

Conclusions: The measurement of salivary cotinine by immunochromatographic assay using NicAlert saliva test is a useful and convenient method for studying the nicotine dependence in tobacco chewers. Immunochromatography-based cotinine test strips are an easy method of detecting salivary cotinine in a dental setup. From this study we are of the opinion that a simple questionnaire like Fagerstorm test can give a less adequate analysis of patient's dependence especially in countries like India, where patients tend to under-report their dependency. Immediate feedback from a chairside test can help both the dentists and patients during a tobacco cessation programme. (J Cancer Prev 2015;20:159-163)
\end{abstract}

Key Words: Immunochromatography, Dependence, Cotinine, Tobacco chewing

\section{INTRODUCTION}

Smokeless tobacco is the non-combusted tobacco that can be used orally or nasally. Oral smokeless tobacco products are placed in the oral cavity and the contents are either sucked or chewed. ${ }^{1}$ In India, smokeless tobacco is used mostly in the form of gutka, which is a combination of crushed areca nut (also called betel nut), tobacco, catechu, paraffin, slaked lime, and sweet or savory flavorings. ${ }^{2}$

According to National Family Health Survey, India-3 (2005-06), in India, $36.5 \%$ males and $8.4 \%$ female in the age group of 12 to 60 years have been found to be consuming smokeless tobacco. ${ }^{3}$

Nicotine is the primary addictive component in tobacco products that acts on the brain. Cotinine is the major metabolite of nicotine and results from the metabolism of nicotine by the cytochrome 2A6 enzyme system in the liver. It is the current marker of choice for the absorption of tobacco exposure because of its relatively long half-life (ten times longer than that of nicotine) ${ }^{3,4}$ Cotinine measurements from human body fluids can provide an assessment of recent exposure to tobacco, but they do not indicate the duration of exposure. Cotinine has been isolated in plasma, urine, saliva and gingival crevicular fluid. ${ }^{5}$ Saliva was

Received May 8, 2015, Revised June 5, 2015, Accepted June 5, 2015

Correspondence to: Dhanya M

Department of Oral Medicine and Radiology, The Oxford Dental College and Hospital, 10th Milestone, Hosur Rd, Bommanahalli, Bengaluru, Karnataka 560068, India

Tel: +91-41716249, Fax: +91-25734656, E-mail: dhanya.mukesh1@gmail.com

Copyright (C) 2015 Korean Society of Cancer Prevention

(c) This is an Open Access article distributed under the terms of the Creative Commons Attribution Non-Commercial License (http://creativecommons.org/licenses/by-nc/4.0) which permits unrestricted non-commercial use, distribution, and reproduction in any medium, provided the original work is properly cited. 
used in our study as it is non-invasive and it is easy to collect without causing much discomfort to the patients.

Tobacco exposes the oral cavity to toxic carcinogens that may have a role in initiation and promotion of cancer. Tobacco is the major etiologic agent causing oral squamous cell carcinoma and is considered to be responsible for $50 \%$ to $90 \%$ of oral cancer cases worldwide. Oral physicians are the first to identify any addictive habits like tobacco and document their ill effects in oral mucosa. So it is important that we take a holistic approach and promote tobacco cessation. The dental practice setting provides a unique opportunity to assist tobacco users in achieving tobacco abstinence.

A precise estimate of tobacco consumption and nicotine dependence in people is an important concern in cessation programmes. In most investigations, tobacco exposure is examined exclusively via a selfadministered questionnaire, the validity of which is often questioned because of underestimation. Self-reported estimates of tobacco use can be less sensitive and reliable. This is particularly true for socially undesirable behaviours or when there are laws banning certain behaviours.

A quantitative assessment of tobacco exposure through evaluation of its metabolites would help overcome such drawbacks. Hence, the main objective of our study was to assess and correlate self-reported nicotine dependence with salivary cotinine content in tobacco chewers. This is one of the first study verifying biochemically the dependence of nicotine in tobacco chewers semi-quantitavely in an institutional dental set-up.

\section{MATERIALS AND METHODS}

The study was approved by the institutional ethical committee as part of a tobacco cessation programme.

\section{Materials}

1) Source of data collection

Seventy-five patients with tobacco chewing habit attending the out-patient department of The Oxford Dental College.

2) Inclusion criteria

Patients aged above 20 years. Healthy patients with a positive history of chewing tobacco alone or in a quid for more than 3 months.

3) Exclusion criteria

Patients with any systemic diseases. Patients with other addictive habits like alcohol and smoking.
Table 1. Cotinine concentration and its interpretation for each level of the NicAlert ${ }^{\mathrm{TM}}$ test

\begin{tabular}{cc}
\hline Level 1 & Cotinine concentration $(\mathrm{ng} / \mathrm{mL})$ \\
\hline 0 & $0-10$ \\
1 & $10-30$ \\
2 & $30-100$ \\
3 & $100-200$ \\
4 & $200-500$ \\
5 & $500-1,000$ \\
6 & $>1,000$ \\
\hline
\end{tabular}

\section{Study design}

A comparative correlation study was planned between nicotine dependence and salivary cotinine level. Following the selection of subjects, a written informed consent was obtained.

Each patient was assessed for nicotine dependence by using Fagerstorm Test for Nicotine dependence-smokeless tobacco (FTND-ST) questionnaire. Cotinine levels in saliva of study group were measured following the guidelines of the users' instruction manual of NicAlert ${ }^{\mathrm{TM}}$ (Nymox Pharmaceutical Corporation, St. Laurent, QC, Canada).

1) Collection of saliva specimens

Unstimulated whole saliva was collected by asking the patient to drool the saliva. The participants' saliva was directed through a small funnel into the collection tube (both included in the NicAlert ${ }^{\mathrm{TM}}$ kit) until the tube was half full. The funnel was then discarded and the tube was capped.

2) Assessment of salivary cotinine

Cotinine levels were measured by NicAlert ${ }^{\mathrm{TM}}$ which is based on the principle of enzymelinked immunosorbent assay (ELISA). In this assay, mouse monoclonal antibody coated gold particles were used with a series of avidity traps that allows quantification. The distance that gold migrates on the strip was known by a clear colour change and provided an accurate measure of the amount of cotinine in the saliva sample. The test strip displayed seven zones with each zone representing a range of level of cotinine. The results were recorded as values from 0 to 6 as shown in Table 1 .

\section{Statistical analysis}

Data were analysed using statistical software (SPSS ver. 12 for Windows; SPSS Inc., Chicago, IL, USA). The chi-square test was used for testing intra-group variation, analysis of variance was used for testing between-groups variation, and Spearman's coefficient was used for measuring the association between variables. 
Table 2. Association between levels of dependency with salivary cotinine levels

\begin{tabular}{lrllll}
\hline \multirow{2}{*}{ Factor } & \multicolumn{3}{c}{ Level of dependency } & \multirow{2}{*}{ Total } \\
\cline { 2 - 4 } & \multicolumn{1}{c}{ Low } & \multicolumn{1}{c}{ Medium } & \multicolumn{2}{c}{ High } & \\
\hline Level 0 & $0(0)$ & $2(9.52)$ & $0(0)$ & $2(2.67)$ \\
Level 1 & $11(34.38)$ & $3(14.29)$ & $2(9.09)$ & $16(21.33)$ \\
Level 2 & $6(18.75)$ & $4(19.05)$ & $6(27.27)$ & $16(21.33)$ \\
Level 3 & $4(12.50)$ & $6(28.57)$ & $8(36.36)$ & $18(24.00)$ \\
Level 4 & $10(31.25)$ & $6(28.57)$ & $5(22.73)$ & $21(28.00)$ \\
Level 5 & $1(3.13)$ & $0(0)$ & $1(4.55)$ & $2(2.67)$ \\
Total & $32(100)$ & $21(100)$ & $22(100)$ & $75(100)$ \\
\hline
\end{tabular}

Values are presented as number (\%). Chi-square $=8.793, P=0.552$.

Table 3. Correlation between total scores and salivary nicotine levels by Spearman's rank correlation

\begin{tabular}{lcccc}
\hline Variable & $\begin{array}{c}\text { Valid } \\
(\mathrm{n})\end{array}$ & $\begin{array}{c}\text { Spearman } \\
(\mathrm{r})\end{array}$ & $t$-value & $P$-level \\
\hline $\begin{array}{c}\text { Total scores and } \\
\text { salivary nicotine levels }\end{array}$ & 75 & 0.230 & 2.026 & $0.046 *$ \\
$* P<0.05$. & & & & \\
\hline
\end{tabular}

\section{RESULTS}

The study sample comprised 75 subjects with a mean age of 34.25 years, of whom 73 (97.33\%) were males and 2 (2.67\%) were females.

There was no significant relation between the total score of the Modified Fagerstorm analysis and the different age group and the gender $(P>0.05)$. The salivary cotinine levels found in the entire study sample were as follows: 0 for 2 subjects (2.67\%), 1 for 16 subjects (21.33\%), 2 for 16 subjects (21.33\%), 3 for 18 subjects (24.00\%), 4 for 21 subjects (28.00\%) and 5 for 2 subjects (2.67\%). There was no significant association found with the level of dependence with salivary cotinine (Table 2 ). A weak correlation ( $\mathrm{r}$ $=0.230$ ) between the total score and salivary cotinine was observed (Table 3). Among the different age groups the correlation between the total score and salivary cotinine was more significant in 20- to 29-year-old age group (Table 4).

\section{DISCUSSION}

A precise estimate of tobacco consumption and nicotine dependence in people is an important concern in cessation programmes. Though measures of nicotine dependence have been developed and validated for cigarette smokers, few nicotine dependence measures have been evaluated for clinical use among tobacco chewers. The present study was carried out on adults
Table 4. Correlation between total scores and salivary nicotine levels by Spearman's rank correlation in all categories

\begin{tabular}{lcccc}
\hline \multicolumn{1}{c}{ Variable } & Valid $(\mathrm{n})$ & Spearman $(\mathrm{r})$ & $t$-value & $P$-level \\
\hline Age $(\mathrm{yr})$ & & & & \\
$20-29$ & 32 & 0.368 & 2.171 & $0.037^{*}$ \\
$30-39$ & 21 & 0.215 & 0.963 & 0.347 \\
$\geq 40$ & 22 & 0.111 & 0.503 & 0.620 \\
Sex & & & & \\
$\quad$ Male & 73 & 0.247 & 2.154 & $0.035^{*}$ \\
Female & 2 & - & - & - \\
\hline
\end{tabular}

$* P<0.05$.

aged between 20 to 50 years with tobacco chewing habit for more than 3 months. Based on their age, the subjects were categorised into 3 groups i.e., 20 to 29 group; 30 to 39 group; $\geq 40$ group. There was no significant difference in the mean score of the questionnaire and the age. Based on the answers to the questions, the subjects were categorized into low, medium and highly dependents. There was no significant correlation between age and dependency.

In 1995, Boyle et al. ${ }^{6}$ published two modified versions of the Fagerström Tolerance Questionnaire (FTQ) for measuring nicotine dependence in smokeless tobacco users. They assessed the association of scores on the modified scales with the concentration of salivary cotinine. The scales correlated 0.33 ( $P$ $<0.01)$ and $0.47(P<0.0001)$ with the cotinine concentrations for the 9-item and 10-item scale, respectively. Clinically, nicotine dependence is most often defined using the eight-item FTQ, and its modified sixitem version, the FTND. ${ }^{5}$

Ebbert et al. ${ }^{7}$ modified the FTND for smokeless tobacco users, referred to this scale as the FTND-ST, and evaluated its characteristics in a population of 42 smokeless tobacco users. The correlation between the FTND-ST total score and the serum cotinine concentrations was $0.53(P<0.001)$. They also concluded that the total score and Items 2, 3 and 5 on the FTND-ST can distinguish between smokeless tobacco use behaviors indicative of higher nicotine exposure. ${ }^{7}$ Our study was in accordance with this study but we have correlated nicotine dependence with salivary cotinine as it was easy to collect and simple to execute in a chair side set up.

Saliva and blood cotinine levels are highly correlated, with a ratio of 1.1 to 1.4 for saliva to blood. An investigation by Bernert et $\mathrm{al}^{8}{ }^{8}$ reported that the serum levels of cotinine were more closely correlated with unstimulated saliva ( $>$ by $4 \%$ ) than with stimulated saliva ( $>$ by $41 \%$ ). Hence, unstimulated salivary cotinine levels were assessed in the current study.

A similar study was done by Nuca et $\mathrm{al}^{5}$ to evaluate the salivary 
cotinine levels and self-reported smoking status in 3- to 44-year-old from Constanta, and also the nicotine dependence in those identified as constant smokers, using the heaviness of smoking index (HSI). In their study they found that there was a significant correlation between the cotinine levels and the selfreported smoking status $(P<0.05)$ and also between cotinine levels and HSI. Our study was similar to this study but the patients included were tobacco chewers; as tobacco chewing is one the leading causes for oral cancer especially in a country like India.

Another similar study was conducted by Balhara et al. ${ }^{9}$ for the assessment of accuracy of self-reported tobacco use among male psychiatric out-patients. The results suggested no significant concordance between the self-reported tobacco use and urinary cotinine levels for both smoking and smokeless tobacco forms. A statistically significant negative correlation between the FTND-smoking form and urine cotinine levels $(r=-0.59)$ and no correlation between FTND-ST scores and urine cotinine levels $(r$ $=-0.08$ ) were observed.

Cotinine is a major metabolite of nicotine, and its concentration in body fluids is determined by the rate of nicotine metabolism and cotinine clearance. Although individual variability might exist in salivary cotinine levels because of these parameters, it is still an important indicator of nicotine dependence. ${ }^{10}$ Determining the concentration of nicotine and cotinine in biological fluids is widely practiced in both epidemiological and clinical smoking studies. Both nicotine and cotinine concentrations are used to estimate tobacco consumption, to determine exposure to environmental smoke and to validate abstinence in smoking cessation programmes. Cotinine, the major metabolite of nicotine, is widely used for estimating exposure to nicotine. This pharmacologically inactive compound has a half-life of 20 hours (15-40 hours), is slowly cleared from the body and is specific to tobacco. ${ }^{11}$

This study assessed the salivary cotinine content and it was correlated to the nicotine dependency assessed by Modified Fagestorm Test for smokeless tobacco. Unlike past ST studies which were particularly undertaken by the of public health researches, to our knowledge this is one of the first study undertaken by oral physicians to assess the salivary cotinine in a simple chair-side method.

Cotinine has been measured in the biologic matrices using various methods like chromatography, colorimetry, raidoimmunoassay, and ELISA, etc. ${ }^{12}$ ELISA with monoclonal antibodies has been proven useful in detecting cotinine in saliva. Studies of ELISA tests for urine cotinine showed almost total agreement with the confirmatory tests like gas chromatography. The NicAlert cotinine test strips (NCTS) used in this study contain cotininespecific monoclonal antibodies and is an immunochromatographic strip that works on the principle of ELISA. It is designed to provide a semiquantitative estimate and can be assessed conveniently by nonspecialists, based solely on visual inspection. Studies by Cooke et al. ${ }^{13}$ have shown that NCTS are a valid alternative to the more expensive and time consuming gas chromatographic tests when testing saliva to verify tobacco exposure. NCTS detect exposure to nicotine from all sources. NCTS have a specificity of $95 \%$, sensitivity of $93 \%$, positive predictive value of $95 \%$, and a negative predictive value of $93 \%$.

Cotinine is a biomarker that has been used to measure dependence among tobacco users. ${ }^{13}$ In the present study salivary cotinine were measured in tobacco chewers and it was correlated with nicotine dependence. A non-significant association was found between the levels of dependence and levels of salivary cotinine. A weak positive correlation was found between total score and salivary cotinine levels $(r=0.230)$. Biochemical assessment of self-reported tobacco use using salivary cotinine by immunochromatographic assay found that patients were underreporting tobacco use.

Evidence from the present study suggests that the accuracy of self-report of tobacco use was found to be low when it was cross checked with salivary cotinine levels. Oral physicians can play a major role in helping subjects quit the habit by making them aware of their self-reported dependence and the cotinine content. This may be considered a baseline value to help in further quitting tobacco habit during a cessation.

The results of this study suggest that biochemical validation of self-reported tobacco use should be done in any tobacco cessation. It is recommended to use biological markers such as salivary cotinine levels to corroborates the information provided by the patients.

Immunochromatographybased cotinine test strips are a relatively easy method for quantification of salivary cotinine chairside. Self-reported tobacco use among people can underestimate the actual prevalence of tobacco use especially in dental patients. Biochemical validation of self-reports is particularly recommended for intervention studies where cessation outcomes are to be measured. As the habit of quitting tobacco is gradual, cotinine test strips can serve as simple, regular, and chairside reminders in a dental office. Biochemical validation of self-reported tobacco use should be considered during prevention and cessation studies among dental patients in developing countries like India. 


\section{CONFLICTS OF INTEREST}

No potential conflicts of interest were disclosed.

\section{REFERENCES}

1. Hatsukami DK, Ebbert JO, Feuer RM, Stepanov I, Hecht SS. Changing smokeless tobacco products new tobacco-delivery systems. Am J Prev Med 2007:33:S368-78.

2. Kumar S, Pandey U, Bala N, Tewar V, Oanh KT. Tobacco habit in northern India. J Indian Med Assoc 2006;104:19-22, 24.

3. Joshi U, Modi B, Yadav S. A study on prevalence of chewing form of tobacco and existing quitting patterns in urban population of Jamnagar, Gujarat. Indian J Community Med 2010;35:105-8.

4. Boffetta P, Hecht S, Gray N, Gupta P, Straif K. Smokeless tobacco and cancer. Lancet Oncol 2008;9:667-75.

5. Nuca CI, Amariei CI, Badea VV, Zaharia AN, Arendt CT. Salivary cotinine, self-reported smoking status and heaviness of smoking index in adults from Constanta, Romania. Oral Health Dent Manag 2011;10:22-31.

6. Boyle RG, Jensen J, Hatsukami DK, Severson HH. Measuring dependence in smokeless tobacco users. Addict Behav 1995;20:44350 .
7. Ebbert JO, Patten CA, Schroeder DR. The Fagerström test for nicotine dependence-smokeless tobacco (FTND-ST). Addict Behav 2006:31:1716-21.

8. Bernert JT Jr, McGuffey JE, Morrison MA, Pirkle JL. Comparison of serum and salivary cotinine measurements by a sensitive high-performance liquid chromatography-tandem mass spectrometry method as an indicator of exposure to tobacco smoke among smokers and nonsmokers. J Anal Toxicol 2000;24:333-9.

9. Balhara YP, Jain R, Sundar AS, Sagar R. Use of cotinine urinalysis to verify self-reported tobacco use among male psychiatric out-patients. Lung India 2012;29:217-20.

10. Jarvis MJ, Fidler J, Mindell J, Feyerabend C, West R. Assessing smoking status in children, adolescents and adults: cotinine cut-points revisited. Addiction 2008;103:1553-61.

11. Robson N, Bond AJ, Wolff K. Salivary nicotine and cotinine concentrations in unstimulated and stimulated saliva. Afr J Pharm Pharmacol 2010;4:61-5.

12. Surya C, Swamy DN, Chakrapani S, Kumar SS. Chairside quantitative immunochromatographic evaluation of salivary cotinine and its correlation with chronic periodontitis. J Indian Soc Periodontol 2012;16:508-12.

13. Cooke F, Bullen C, Whittaker R, McRobbie H, Chen MH, Walker N. Diagnostic accuracy of NicAlert cotinine test strips in saliva for verifying smoking status. Nicotine Tob Res 2008;10:607-12. 